\title{
Communication \\ Electrodeposited Copolymer Films with Tunable Conductivity
}

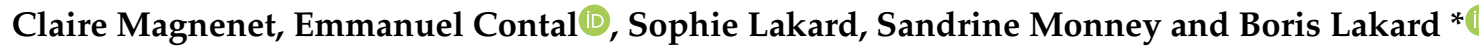 \\ Institut UTINAM UMR CNRS 6213, UFR Sciences et Techniques, University Bourgogne Franche-Comté, \\ 16 Route de Gray, 25030 Besançon, France; claire.magnenet@univ-fcomte.fr (C.M.); \\ emmanuel.contal@univ-fcomte.fr (E.C.); sophie.lakard@univ-fcomte.fr (S.L.); \\ sandrine.monney@univ-fcomte.fr (S.M.) \\ * Correspondence: boris.lakard@univ-fcomte.fr; Tel.: +33-381662046
}

Received: 4 September 2020; Accepted: 7 October 2020; Published: 12 October 2020

\begin{abstract}
Conducting copolymer films were prepared from pyrrole (Py) and 1,12-di-(1-pyrrolyl) dodecane (DiPy) in an attempt to prepare conducting films that can be used as sensitive material of chemiresistor gas sensors. Copolymer thin films were obtained by electrochemical oxidation in a lithium perchlorate/acetonitrile electrolyte with different feed ratios of comonomers. Increasing the portion of DiPy in the comonomer mixture resulted in the formation of thinner and less rough copolymer films and to a modification of their morphology from a granular structure to a clover-like structure. In addition, copolymer films with very different conductivities were obtained by varying the comonomers ratio. Indeed, the conductivity of the copolymer containing $91 \%$ of Py was $2 \times 10^{5}$ times higher than the conductivity of the polymer containing $91 \%$ of DiPy, indicating that it is possible to tune the conductivity of the film by varying the composition of the initial comonomer mixture.
\end{abstract}

Keywords: conducting polymer; electrodeposition; copolymerization; surface analysis

\section{Introduction}

The discovery of polyacetylene [1] has generated a great interest for electrically conducting organic polymers such as polypyrroles, polyanilines, or polythiophenes [2,3]. The most outstanding property of this class of materials comes from the ease to electrochemically control and modulate their doping level to reversibly switch from an insulating to a metallic state [4]. Among heterocyclic conducting polymers, considerable attention has been drawn to electrodeposited polypyrrole and its derivatives because of their high conductivity, thermal and environmental stability, and ease of electrosynthesis $[5,6]$. To date, polypyrrole films have been used for various applications including: batteries and energy storage [7-9], tissue engineering [10], drug delivery [11], cytometry [12,13], and biosensors [5].

To overcome these drawbacks, a strategy consists of the copolymerization of pyrrole with other monomers since copolymerization offers the possibility to modify the physico-chemical properties of the prepared films. This was done by Massoumi et al. who copolymerized pyrrole with $N$-ethyl, $\mathrm{N}$-butyl, and $\mathrm{N}$-octyl pyrrole with various monomer ratios and demonstrated that the conductivity of the copolymers was inversely correlated with the alkyl chain length due to more or less planar structure [14]. Similarly, Cihaner et al. electro-oxidized a solution containing pyrrole and monomers with two pyrrolyl groups linked by a polyether spacer $[15,16]$. This oxidation led to the formation of $N$-substituted polyethereal polypyrrole films whose conductivity increased when the portion of pyrrole in the comonomer mixture was increased. In addition, Jang et al. successfully copolymerized poly(3,4-ethylenedioxythiophene) and poly(3-hexylthiophene) in the vapor phase to give tunable conductivity by varying the comomer ratio [17]. Thus, depending on the composition of the electrolyte, 
the electrical conductivity changed in the range of $3.0 \times 10^{-2}-2.3 \times 10^{1} \mathrm{~S} / \mathrm{cm}$. Similarly, Waware et al. prepared poly(aniline-co-3-nitroaniline) films with varying compositions obtained by in situ oxidative copolymerization of aniline and 3-nitroaniline by altering the molar ratio of monomers [18]. These films exhibited electrical conductivity comprised between $1.53 \times 10^{-6}$ and $3.46 \times 10^{-6} \mathrm{~S} / \mathrm{cm}$.

A similar strategy is used here since we seek to prepare copolymer films with tunable conductivity by electropolymerization of pyrrole and 1,12-di-(1-pyrrolyl)dodecane monomers. Pyrrole was chosen due to the high conductivity of polypyrrole films, which can reach up to $150 \mathrm{~S} / \mathrm{cm}$ [19], when DiPy was chosen because it contains a spacer that may decrease the conductivity of the copolymer by breaking the conjugation in the polymer backbone. Therefore, it is expected that such copolymerization from monomers with very different electrical structure may lead to strongly different conductivities.

\section{Materials and Methods}

\subsection{Materials and Reagents}

Pyrrole (>98\%, CAS: 109-97-7) was purchased from Acros (Illkirch, France) and distilled under reduced pressure before use. Acetonitrile (>99.5\%, CAS: 75-05-8) was purchased from Fisher Scientific (Illkirch, France) and used as a solvent for electrochemical experiments. Lithium perchlorate (>95\%, CAS: 7791-03-9) was purchased from Sigma Aldrich (St. Quentin Fallavier, France) and used as supporting salt for electrochemical experiments. Rectangular-shaped Fluorine-doped Tin Oxide (FTO) substrates ( $15 \mathrm{~mm} \times 30 \mathrm{~mm}$, thickness: $1.1 \mathrm{~mm}, \mathrm{R}=80 \Omega$ /square) were produced by Asahi and purchased from Solems (Palaiseau, France).

\subsection{Synthesis of 1,12-di-(1-Pyrrolyl)dodecane (DiPy)}

Pyrrole (4.02 g, $60 \mathrm{mmol}, 3 \mathrm{eq})$ was added to a cooled solution $\left(5^{\circ} \mathrm{C}\right)$ of sodium hydride (60\% wt, in mineral oil, Fisher, CAS: 7646-69-7) (2.00 g, $50 \mathrm{mmol}, 2.5 \mathrm{eq})$ in dry dimethylformamide (100 mL, Fisher, 99.8\% over molecular sieve, CAS: 68-12-2). The mixture was allowed to reach room temperature and stirred for $30 \mathrm{~min}$ then cooled again to $0{ }^{\circ} \mathrm{C}$. 1,12-dibromododecane (6.52 g, $20 \mathrm{mmol}, 1 \mathrm{eq}$, Alfa Aesar, 97\%, CAS: 4101-68-2) was dissolved at $0{ }^{\circ} \mathrm{C}$ in $100 \mathrm{~mL}$ of dry dimethylformamide. The mixture was allowed to reach room temperature and stirred for $24 \mathrm{~h}$. The resulting solution was hydrolyzed by addition of water and the product was extracted with ethyl acetate $(3 \times 100 \mathrm{~mL}$, Fisher, 99\%, CAS: 141-78-6). The resulting organic phase was washed with water $(3 \times 100 \mathrm{~mL})$, dried over sodium sulfate (Roth $99 \%$, CAS: 7757-82-6), filtered and the solvent was removed under vacuum. The crude product was purified by silica gel chromatography (eluent: dichloromethane/petroleum ether, 1:9). The desired product was isolated as a pale yellow oil $(4.80 \mathrm{~g}, 80 \%)$.

${ }^{1} \mathrm{H}$ NMR $\left(400 \mathrm{MHz}, \mathrm{CDCl}_{3}\right): \delta=6.66(\mathrm{~s}, 4 \mathrm{H}), 6.15(\mathrm{~s}, 4 \mathrm{H}), 3.87(\mathrm{t}, \mathrm{J}=7.2 \mathrm{~Hz}, 4 \mathrm{H}), 1.78(\mathrm{~m}, 4 \mathrm{H})$, $1.29(\mathrm{~m}, 16 \mathrm{H}) .{ }^{13} \mathrm{C}$ NMR $\left(100.6 \mathrm{MHz}, \mathrm{CDCl}_{3}\right): \delta=120.6,107.8,49.7,31.7,29.6,29.6,29.3,26.9 \mathrm{ppm}$.

\subsection{Electrochemical Deposition of Polymer Films}

Electrochemical experiments were carried out at room temperature in a three-electrode and single compartment glass cell. A platinum wire, with an area of $0.785 \mathrm{~mm}^{2}$, or a FTO plate, with an area of $1.5 \mathrm{~cm}^{2}$, was used as the working electrode. A platinum sheet and a Saturated Calomel Electrode (SCE) were used as the counter and reference electrodes, respectively. Cyclic voltammograms (CVs) and chronoamperometries (CAs) were recorded by a VersaSTAT MC potentiostat/galvanostat from Princeton Applied Research.

The electrolytic solutions were mixtures of pyrrole and 1,12-di-(1-pyrrolyl)dodecane monomers. The molar ratios of Py to DiPy (Py:DiPy) were selected to be 91:9, 83:17, 50:50, 17:83, and 9:91. For example, to obtain a Py:DiPy ratio of 91:9, $138 \mathrm{mg}$ of Py $(0.1 \mathrm{M})$ and $73 \mathrm{mg}$ of DiPy $(0.01 \mathrm{M})$ were dissolved in $20 \mathrm{~mL}$ of an acetonitrile solution containing $0.1 \mathrm{M} \mathrm{LiClO}_{4}$. 
Then, the different electrolytic solutions were electrochemically copolymerized onto a platinum wire by applying 5 potential sweeps between 0 and $+2 \mathrm{~V} / \mathrm{SCE}$ with a scan rate of $50 \mathrm{mV} / \mathrm{s}$. The resulting cyclic voltammetries were used to analyze the copolymerization process.

In addition, the different electrolytic solutions were electrochemically copolymerized onto an FTO plate by applying a potential of $+1.5 \mathrm{~V} / \mathrm{SCE}$ during $180 \mathrm{~s}$. The resulting copolymer films were then rinsed with acetonitrile and their properties were characterized. The chronoamperometry curves were used to analyze the copolymerization process and estimate the thickness of the copolymer films.

\subsection{Characterization Techniques}

Mechanical Profilometry. The thickness and roughness of each copolymer film were measured using an Alpha-Step IQ stylus-based surface profiler from KLA Tencor. Arithmetic roughness (Ra) was measured on a scan length of $2000 \mu \mathrm{m}$ at a scan speed of $50 \mu \mathrm{m} \cdot \mathrm{s}^{-1}$. Thickness (T) was measured in the same operating conditions after creation of a physical sharp step in the film. Reported Ra and $\mathrm{T}$ are the mean of at least 5 measurements done at different places of the samples.

SEM microscopy. The surface topography of each copolymer film was imaged, without prior metallization, using a high-resolution SEM microscope Quanta $450 \mathrm{~W}$ from FEI. The electron beam energy used was $10 \mathrm{keV}$ and the working distance used was $10 \mathrm{~mm}$.

AFM microscopy. The surface topography and resistance mapping of each copolymer film were obtained using a Nano Observer AFM microscope from CSI. In particular, a resistance mapping of the films was achieved using the Resiscope mode of the AFM instrument. Thus, the resistance was measured by applying a DC bias between the copolymer film and a conductive AFM probe whose tip was scanning in contact mode using the laser deflection for the AFM feedback. Silicon probes coated with Pt-Ir on both sides (ANSCM-PT probes, from AppNano) and silicon cantilevers (225 $\mu \mathrm{m}$ long, with a spring constant of $1-5 \mathrm{~N} \cdot \mathrm{m}^{-1}$ ) were used for AFM measurements. AFM picture treatment was done using Mountains Map ${ }^{\circledR}$ SPM software from Digital Surf.

All of these characterization experiments were applied to copolymer films obtained by chronoamperometry on FTO substrates.

\section{Results and Discussion}

This section may be divided by subheadings. It should provide a concise and precise description of the experimental results, their interpretation as well as the experimental conclusions that can be drawn.

\subsection{Electrochemical Polymerization of Py:DiPy Mixtures}

\subsubsection{Cyclic Voltammetry}

First, 1,12-di-(1-pyrrolyl)dodecane monomers were synthesized using the protocol described in Supporting Information. Briefly, pyrrole and 1,12-dibromododecane were added in dimethylformamide, the solution was then stirred and hydrolyzed. The resulting product (DiPy) was extracted, washed, filtered, and purified leading to the formation of a pale yellow oil characterized using ${ }^{1} \mathrm{H}$ and ${ }^{13} \mathrm{C}$ NMR. Electrochemical oxidation of DiPy was then performed in $0.1 \mathrm{M} \mathrm{LiClO}_{4}$ /acetonitrile solution using cyclic voltammetry (CV). During the first potential scan, the anodic oxidation of DiPy monomers leads to a broad irreversible oxidation peak appearing at $+1.4 \mathrm{~V} / \mathrm{SCE}$ (Figure 1a). Such broad irreversible peak has already been observed with other pyrrole modified with a long alkyl chain-for example $N$-undecylpyrrole and Pyrrole- $N$-undecanoic acid [20]. This peak corresponds to the oxidation of DiPy monomers into radical cations which initiates the polymerization reaction. However, this anodic peak loses strongly in intensity and nearly disappears from the second potential scan indicating that the electropolymerization process rapidly slows down. A strong oxidation peak is also visible during the anodic oxidation of Py monomers at $+1.45 \mathrm{~V} / \mathrm{SCE}$ (Figure 1d), but this peak is still present after each repetitive cycle indicating that the electrodeposition of a conducting polypyrrole film takes place at the Pt electrode. 


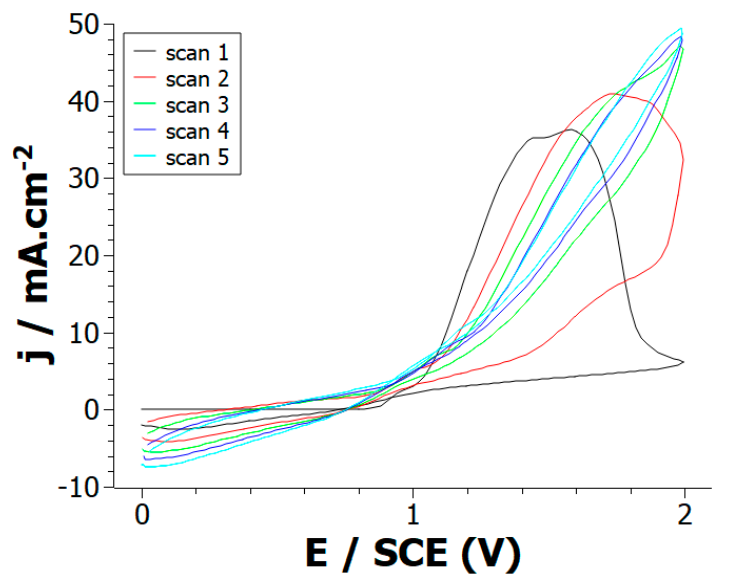

(a)

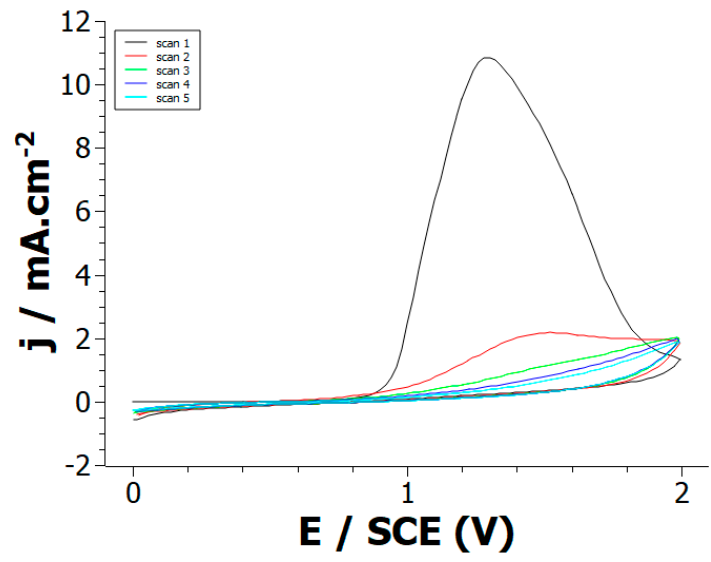

(c)

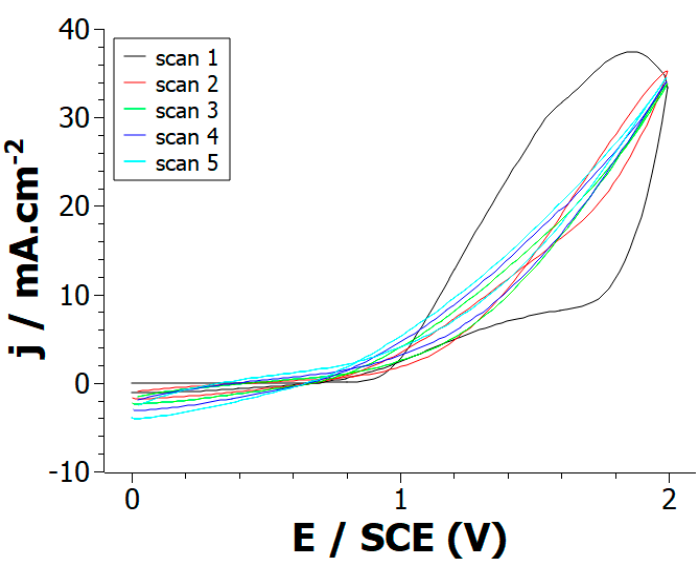

(b)

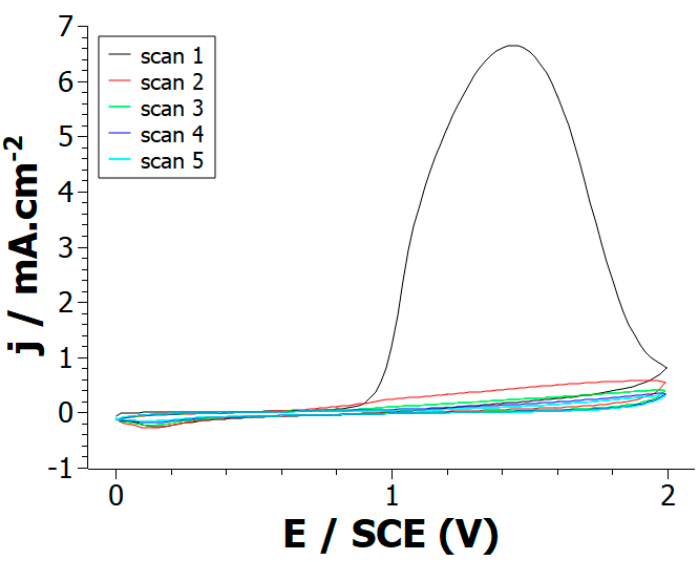

(d)

Figure 1. Cyclic voltammograms of: (a) Py, (b) Py:DiPy 50:50 mixture, (c) Py:DiPy 9:91 mixture, (d) DiPy in acetonitrile solutions containing $0.1 \mathrm{M} \mathrm{LiClO}_{4}$. Repetitive cycling between 0 and $+2 \mathrm{~V} / \mathrm{SCE}$ at a scan rate of $50 \mathrm{mV} / \mathrm{s}$ on a Pt electrode.

The electropolymerization mechanism of pyrrole and $N$-substituted pyrroles is well-known and has been deeply studied [21]. The polymerization reaction starts with the oxidation of monomer surface of the electrode to form a cation radical which dimerizes in the $\alpha$-position (Figure 2). The coupling between two radicals results in the formation of a bond between their $\alpha$-positions and the formation of the dihydromer dication. The loss of two protons forms the aromatic dimer, then the polymerization reaction continues with the oxidation of the dimer into the corresponding cation radical and the same steps take place until formation of a polypyrrole film at the electrode surface.

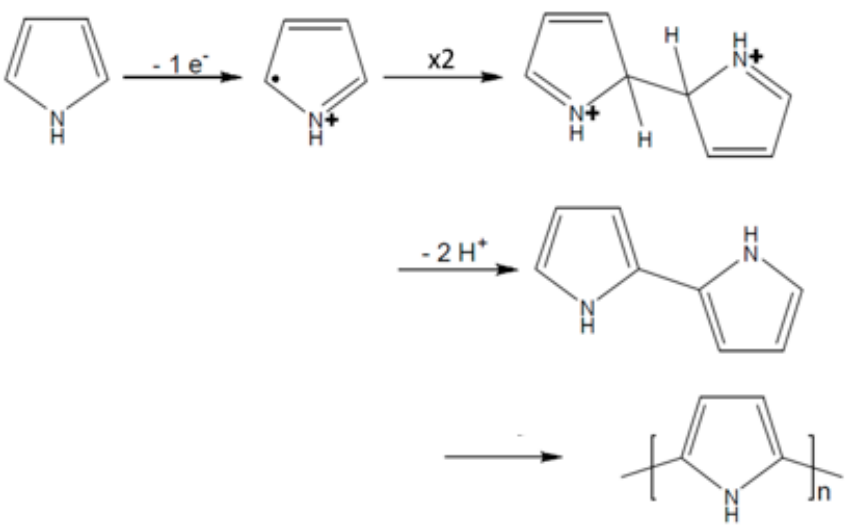

Figure 2. Mechanism of pyrrole electropolymerization. 
If the mechanism of DiPy has never been established before, it is likely to follow the same steps as the mechanism of pyrrole and $N$-substituted pyrroles.

$$
2 \mathrm{Py}-\left(\mathrm{CH}_{2}\right)_{12}-\mathrm{Py} \stackrel{-2 e^{-}}{\rightarrow} \stackrel{-2 \mathrm{H}^{+}}{\rightarrow} \mathrm{Py}-\left(\mathrm{CH}_{2}\right)_{12}-\mathrm{Py}-\mathrm{Py}-\left(\mathrm{CH}_{2}\right)_{12}-\mathrm{Py} \stackrel{-2 n e^{-}}{\rightarrow} \stackrel{2 \mathrm{nH}^{+}}{\rightarrow}\left(\mathrm{Py}-\left(\mathrm{CH}_{2}\right)_{12}-\mathrm{Py}\right)_{\mathrm{n}-1}
$$

These experiments prove that Py and DiPy have a very close oxidation potential, so the formation of copolymers by anodic oxidation of monomer mixtures may be feasible. Therefore, $\mathrm{CV}$ s were recorded from solutions containing various ratios of Py and DiPy. The CVs recorded for the Py:DiPy ratios of 91:9, 83:17 and 50:50 (Figure 1c) are very similar and exhibit during the first scan a strong anodic peak whose current density is comparable to the one obtained during the oxidation of Py. During the following scans, the copolymerization continues to take place at the Pt electrode since the current density remains high. The CVs obtained for Py:DiPy ratios of 17:83 and 9:91 (Figure 1b) also present a strong anodic peak, but the current density decreases from the second potential scan. Therefore, the electrochemical behavior observed with these ratios is rather similar to the one obtained with DiPy even if the intensity of the anodic peak is a little bit higher.

\subsubsection{Chronoamperometry}

To complete these results, copolymer films were deposited by chronoamperometry (CA). In all cases, a short initial increase in current density was observed that is related to the nucleation process associated with the oxidation of Py and/or DiPy monomers into radical cations (Figure 3). After this initial increase, the current density remains stable, at $2.7 \mathrm{~mA} / \mathrm{cm}^{2}$, for Py indicating that the polymerization process takes place without any limitation and leads to the deposition of a conducting film. On the contrary, during the oxidation of DiPy monomers, the initial increase is quickly followed by a very strong decrease of the current density indicating that the electropolymerization process of DiPy monomers is rapidly limited and suggesting that the polymer film formed has a low conductivity. When the oxidation of Py:DiPy mixtures is performed, a decrease of the current density is also observed, but this decrease is slower. Moreover, the current density decrease is less marked when the portion of DiPy monomers is decreased (for Py:DiPy 50:50 from 2.8 to $1.5 \mathrm{~mA} / \mathrm{cm}^{2}$, and for Py:DiPy 9:91 from 2.9 to $0.4 \mathrm{~mA} / \mathrm{cm}^{2}$ ) due to the presence of the alkyl chains in the DiPy monomers which prevents the conjugation in the copolymer backbone.

\subsection{Physico-Chemical Properties of Electrodeposited Py:DiPy Copolymers}

For all Py:DiPy ratios, a black thin solid film is observed onto the working electrode at the end of the CV or CA experiment indicating that the copolymerization has been successful even if different electrochemical behaviors have been observed. The thickness of these copolymer films was measured by profilometry. Both thickness and roughness of the films decrease when the DiPy portion increases (Table 1). For example, a 91:9 ratio leads to a thickness of $6.2 \mu \mathrm{m}$ (and a roughness of $1.4 \mu \mathrm{m}$ ) when a ratio of 9:91 leads to a thickness of $1.1 \mu \mathrm{m}$ (and a roughness of $0.1 \mu \mathrm{m}$ ). This can be explained by the presence of the long alkyl chain spacer which partially prevents the formation of the copolymer by decreasing the delocalization of $\pi$ electrons in the copolymer backbone. Due to this phenomenon, the thickness of each copolymer film is logically lower than the thickness of polyPy $(8.1 \mu \mathrm{m})$ and higher than the one of polyDiPy which is very thin $(<0.2 \mu \mathrm{m})$ due to the steric repulsion interactions of the alkyl groups.

Since chemical structures of comonomers and electrochemical experiments suggest that the conductivity of the copolymer films varies with the Py:DiPy ratio, a resistance mapping of each copolymer film deposited on FTO substrates was achieved, using an AFM microscope with the Resiscope mode, and compared with the resistance mapping of a polypyrrole film (Figure 4). Moreover, for each film, the arithmetic mean resistance was calculated by Mountains Map ${ }^{\circledR}$ software from the resistances measured in each point of the map (Table 1). 


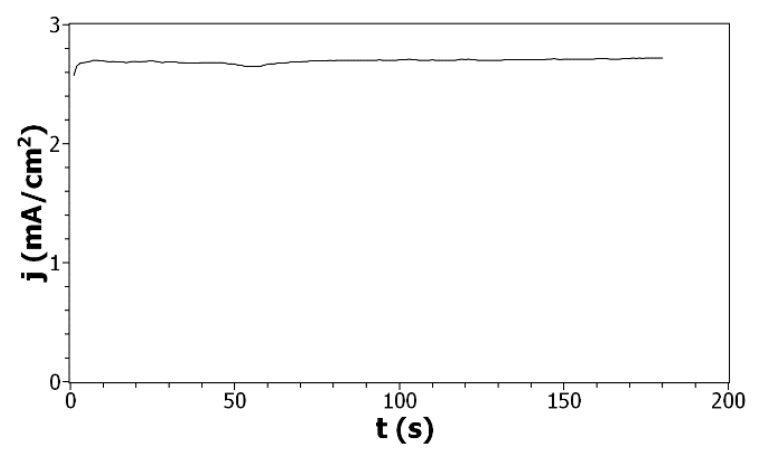

(a)

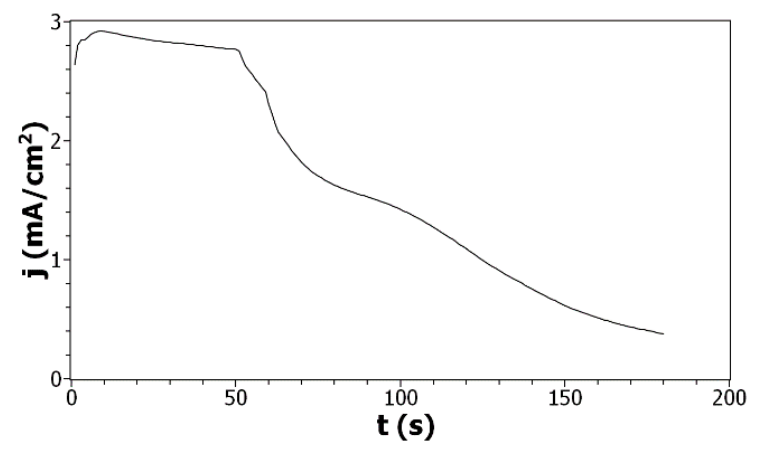

(c)

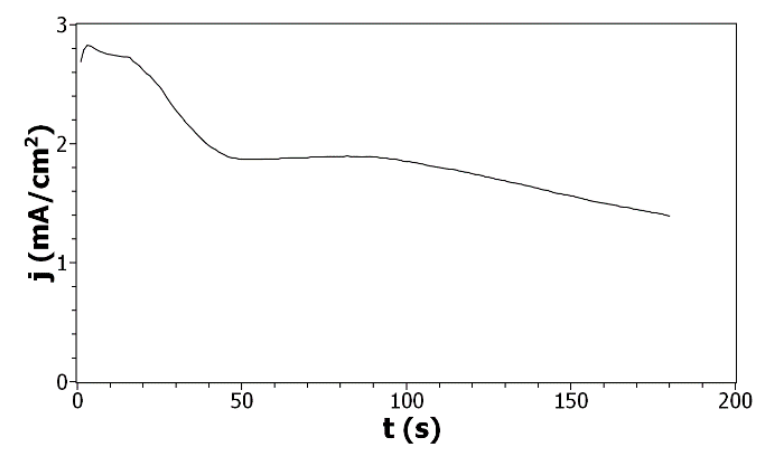

(b)

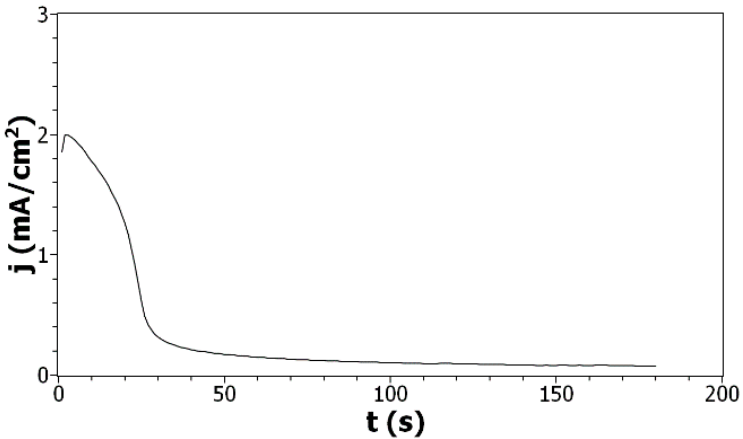

(d)

Figure 3. Current density—time plots of: (a) Py, (b) Py:DiPy 50:50 mixture, (c) Py:DiPy 9:91 mixture, (d) DiPy in acetonitrile solutions containing $0.1 \mathrm{M} \mathrm{LiClO}_{4} . \mathrm{E}=+1.5 \mathrm{~V} / \mathrm{SCE} . \mathrm{t}=3 \mathrm{~min}$. WE $=\mathrm{FTO}$.

Table 1. Resistance (R), thickness (T) and roughness values (Ra) for polyPy, polyDiPy and their copolymers obtained from different mixtures.

\begin{tabular}{cccc}
\hline Py:DiPy Ratio & $\mathbf{R}(\mathbf{M} \boldsymbol{\Omega})$ & $\mathbf{T}(\boldsymbol{\mu m})$ & $\mathbf{R a}(\boldsymbol{\mu m})$ \\
\hline $100: 0$ & $0.025+/-0.013$ & $8.1+/-0.7$ & $1.7+/-0.3$ \\
$91: 9$ & $0.069+/-0.027$ & $6.2+/-0.5$ & $1.4+/-0.2$ \\
$83: 17$ & $52+/-17$ & $4.8+/-0.4$ & $1.2+/-0.2$ \\
$50: 50$ & $74+/-40$ & $3.0+/-0.3$ & $0.7+/-0.1$ \\
$17: 83$ & $779+/-113$ & $1.7+/-0.2$ & $0.20+/-0.05$ \\
$9: 91$ & $13,525+/-5379$ & $1.1+/-0.2$ & $0.10+/-0.05$ \\
$0: 100$ & $169,666+/-4444$ & $<0.2+/-0.1$ & $<0.002+/-0.001$ \\
\hline
\end{tabular}

The resistance map of the polyPy film shows blue spots corresponding to the highest resistance values (>0.04 M $\Omega$ ) and consequently to the less conductive parts of the film (Figure $4 \mathrm{a}$ ). These low conductive spots are randomly distributed in a wide yellow area which corresponds to lower resistance values (0-0.02 M $\Omega$ ). From this map, $R$ can be estimated to only $25 \mathrm{k} \Omega$ indicating that the electrodeposited polypyrrole films are highly conducting. On the contrary, the resistance of the polyDiPy film has a very low conductivity since $\mathrm{R}$ can be estimated to $169,166 \mathrm{M} \Omega$. Considering these measurements, it is not surprising to observe that the resistance increases at the same time as the portion of DiPy monomers increases in the Py:DiPy mixtures (Table 1). It is also noticeable that the changes in resistance with the Py:DiPy ratio is very marked since it increases from $69 \mathrm{k} \Omega$ for the Py:DiPy 91:9 ratio to 13,525 M $\Omega$ for the Py:DiPy 9:91 ratio. The resistance maps of the different copolymers (Figure $4 b-d$ ) show a homogeneous distribution of the resistance values suggesting a uniform and homogeneous structure of the copolymer films which is confirmed by SEM and AFM pictures (Figure 5). Indeed, whatever the Py:DiPy ratio, the surface morphology appears very homogeneous and consists either in micrometric clusters formed by aggregation of small granular nodules (for Py portion $>50 \%$ ) or in bigger clusters 
looking like clovers (for Py portion $<50 \%$ ). The images of polyDiPy are not given since they are not really meaningful due to the low thickness of this film.

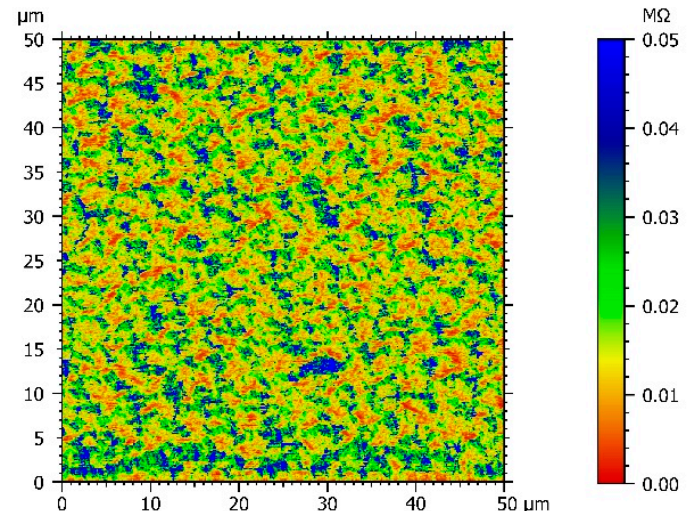

(a)

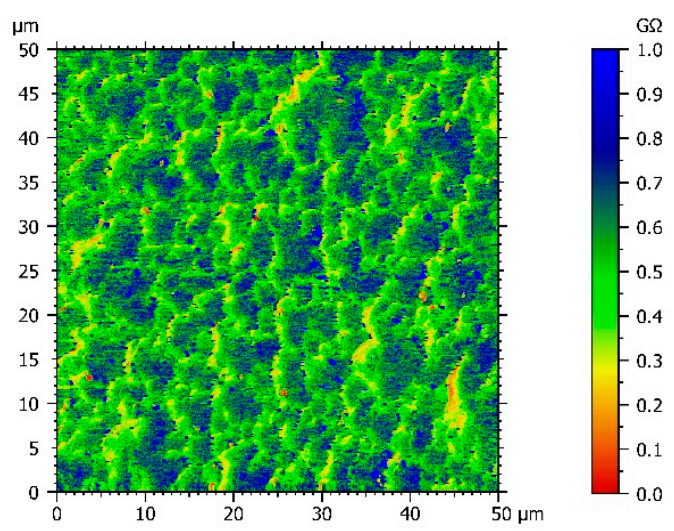

(c)

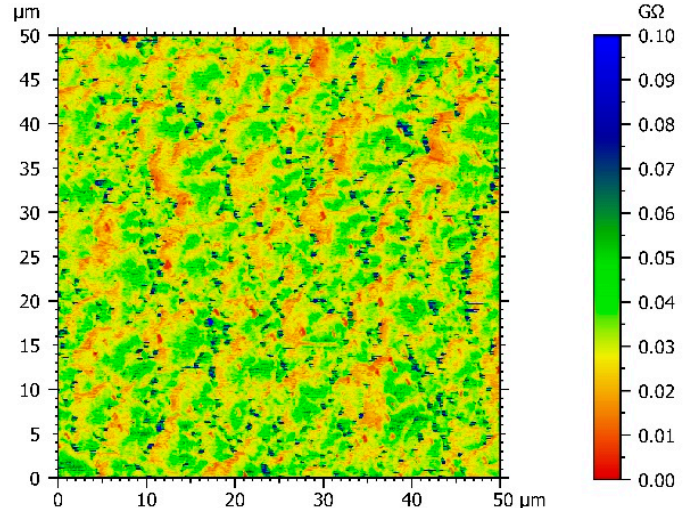

(b)

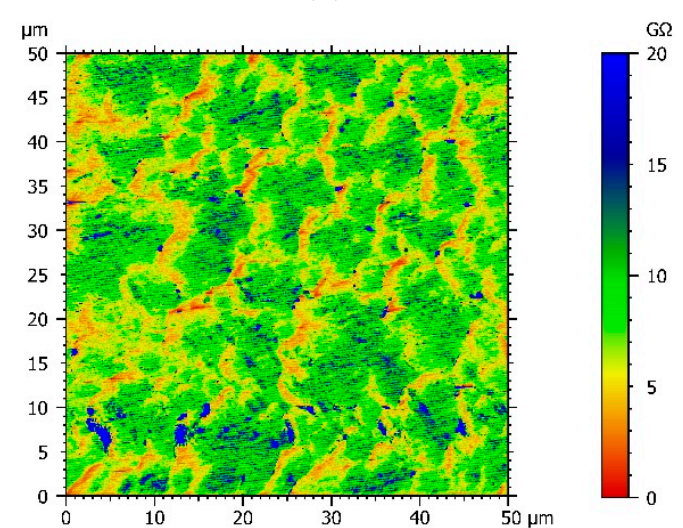

(d)

Figure 4. AFM resistance maps of: (a) Py, (b) Py:DiPy 50:50 mixture, (c) Py:DiPy 17:83 mixture, (d) Py:DiPy 9:91 mixture.

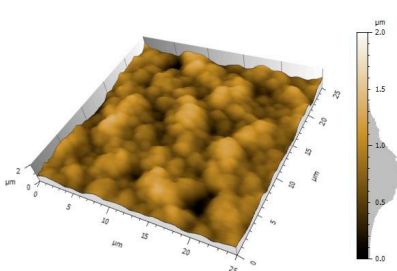

(a)

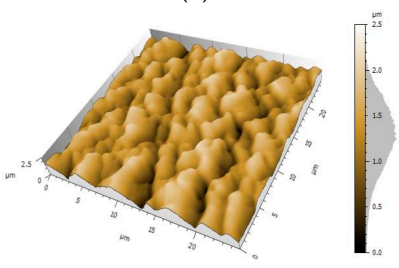

(c)

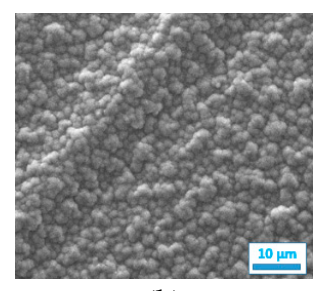

(b)

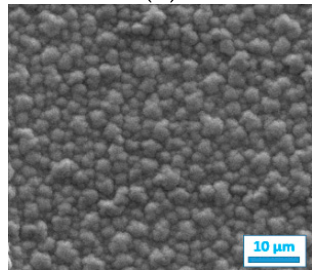

(d)

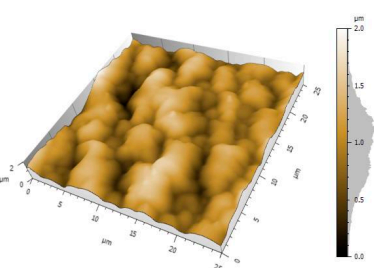

(e)

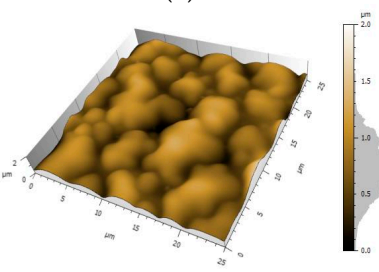

(g)

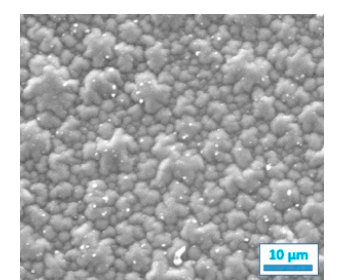

(f)

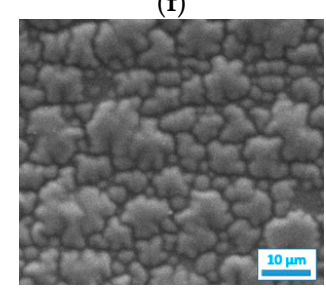

(h)

Figure 5. AFM and SEM images of the films deposited from: (a,b) Py, (c,d) Py:DiPy 91:9 mixture, (e,f) Py:DiPy 50:50 mixture, (g,h) Py:DiPy 9:91 mixture.

Of course, the value of the resistance varied a little bit from one point to the other but such variation is not surprising for a conducting film whose structure is composed of both doped and 
dedoped monomers and also of two different monomers. Moreover, since the AFM resistance maps don't exhibit two separate domains, each containing one of the starting monomers, it can be assumed that the resulting copolymer film consists of a single phase containing the two initial comonomers.

The main reason of the low conductivity of polyDiPy compared to polyPy is the stereochemical differences between these polymers. Indeed, polyPy has a nearly planar structure with a low ionization potential because of the strong delocalization of $\pi$ electrons. For polyDiPy, the ring-ring planarity of the polymer is prevented by steric van der Waals repulsion interactions by alkyl groups which makes the hopping process between chains more difficult and leads to a lower conductivity. Such tendency was already observed by Massoumi et al. during the copolymerization of pyrrole and $N$-alkylpyrroles [5]. The same phenomenon takes place with copolymers since the copolymers obtained from comonomer mixtures containing a high portion of DiPy are less conductive due to the presence of numerous DiPy monomers with long alkyl chains in the film. Indeed, the steric effect of the alkyl spacer on the conductivity increases with the increasing ratio of DiPy in the comonomer mixture, which makes the hopping process between chains more difficult.

\section{Conclusions}

In conclusion, this work establishes a method for preparing copolymer thin films with tunable conductivity. Indeed, by varying the ratio of 1,12-di-(1-pyrrolyl)dodecane and pyrrole monomers in the electrolytic solution, it is possible to obtain copolymer films with different morphology (granular or clover like), thickness, and roughness, as well as resistance going from very low values $(0.0069 \mathrm{M} \Omega$ for a Py:DiPy ratio of 91:9) to very high values (13525 M $\Omega$ for a Py:DiPy ratio of 9:91). Moreover, the conductivity of the copolymer films appears inversely correlated with the portion of DiPy due to steric van der Waals repulsion interactions by alkyl groups which makes the hopping process between polymer chains more difficult. Therefore, this work demonstrates that it is possible to tune the conductivity of the copolymer film by varying the initial ratio of comonomers. This allows for considering the use of these copolymers for electrocatalysis, for the fabrication of batteries, or for the development of liquid and gas sensors. Future work will focus on the incorporation of these copolymer films as sensitive materials in ammonia gas sensors in order to correlate the resistance of the film to the sensor's response characteristics.

Author Contributions: DiPy monomers, E.C.; electrochemical experiments, C.M. and S.L.; AFM measurements, S.M.; SEM analyses and profilometric measurements, B.L. All authors have read and agreed to the published version of the manuscript.

Funding: This research was partly funded by the Bourgogne Franche-Comté Regional Council through the COMICS project. It was also supported by the French RENATECH network and its FEMTO-ST technological facility.

Conflicts of Interest: The authors declare no conflict of interest.

\section{References}

1. Shirakawa, H.; Louis, E.J.; MacDiarmid, C.K.; Chiang, C.K.; Heeger, A.J. Synthesis of electrically conducting organic polymers: Halogen derivatives of polyacetylene, $(\mathrm{CH})_{\mathrm{x}}$. J. Chem. Soc. Chem. Commun. 1977, 16, 578-580. [CrossRef]

2. Naveen, M.H.; Gurudatt, N.G.; Shim, Y.B. Applications of conducting polymer composites to electrochemical sensors: A review. Appl. Mater. Today 2017, 9, 419-433. [CrossRef]

3. Han, Y.; Dai, L. Conducting polymers for flexible supercapacitors. Macromol. Chem. Phys. 2019, 220, 1800355. [CrossRef]

4. Thomas, E.M.; Davidson, E.C.; Katsumata, R.; Segalman, R.A.; Chabinyc, M.L. Branched side chains govern counterion position and doping mechanism in conjugated polythiophenes. ACS Macro Lett. 2018, 7, 1492-1497. [CrossRef]

5. Jain, R.; Jadon, N.; Pawaiya, A. Polypyrrole based next generation electrochemical sensors and biosensors: A review. TrAC Trends Anal. Chem. 2017, 97, 363-373. [CrossRef] 
6. Stejskal, J.; Trchova, M. Conducting polypyrrole nanotubes: A review. Chem. Papers 2018, 72, 1563-1595. [CrossRef]

7. Yuan, T.; Ruan, J.F.; Zhang, W.M.; Tan, Z.P.; Yang, J.H.; Ma, Z.F.; Zheng, S.Y. Flexible overoxidized polypyrrole films with orderly structure as high-performance anodes for li- and na-ion batteries. ACS Appl. Mater. Interfaces 2016, 51, 35114-35122. [CrossRef] [PubMed]

8. Jia, X.T.; Ge, Y.; Shao, L.; Wang, C.; Wallace, G.G. Tunable conducting polymers: Toward sustainable and versatile batteries. ACS Sustain. Chem. Eng. 2019, 7, 14321-14340. [CrossRef]

9. Yang, C.Y.; Zhang, P.F.; Nautiyal, A.; Li, S.H.; Liu, N.; Yin, J.L.; Deng, K.L.; Zhang, X.Y. Tunable three-dimensional nanostructured conductive polymer hydrogels for energy-storage applications. ACS Appl. Mater. Interfaces 2019, 11, 4258-4267. [CrossRef]

10. Tiwari, A.P.; Bhattarai, D.P.; Maharjan, B.; Ko, S.W.; Kim, H.Y.; Park, C.H.; Kim, C.S. Polydopamine-based implantable multifunctional nanocarpet for highly efficient photothermalchemo therapy. Sci. Rep. 2019, 9, 2943. [CrossRef]

11. Tiwari, A.P.; Hwang, Y.I.; Oh, J.M.; Maharjan, B.; Chun, S.; Kim, B.S.; Joshi, M.K.; Park, C.H.; Kim, C.S. $\mathrm{pH} / \mathrm{NIR}$-responsive polypyrrole-functionalized fibrous localized drug-delivery platform for synergistic cancer therapy. ACS Appl. Mater. Interfaces 2018, 10, 20256-20270. [CrossRef] [PubMed]

12. Foysal, Z.K.; Hutcheson, J.A.; Hunter, C.J.; Powless, A.J.; Benson, D.; Fritsch, I.; Muldoon, T.J. Redox-magnetohydrodynamically controlled fluid flow with poly(3,4-ethylenedioxythiophene) coupled to an epitaxial light sheet confocal microscope for image cytometry applications. Anal. Chem. 2018, 90, 7862-7870.

13. Foysal, Z.K.; Fritsch, I. Chip-scale electrodeposition and analysis of poly(3,4-ethylenedioxythiophene) (pedot) films for enhanced and sustained microfluidics using dc-redox-magnetohydrodynamics. J. Electrochem. Soc. 2019, 166, H615-H627.

14. Massoumi, B.; Isfahani, N.S.; Saraei, M.; Entezami, A. Investigation of the electroactivity, conductivity, and morphology of poly(pyrrole-co-N-alkyl pyrrole) prepared via electrochemical nanopolymerization and chemical polymerization. J. Appl. Polym. Sci. 2012, 124, 3956-3962. [CrossRef]

15. Cihaner, A. Electrochemical synthesis of new conducting copolymers containing pseudo-polyether cages with pyrrole. J. Electroanal. Chem. 2007, 605, 8-14. [CrossRef]

16. Mert, O.; Demir, A.S.; Cihaner, A. Pyrrole coupling chemistry: Investigation of electroanalytic, spectroscopic and thermal properties of N-substituted poly(bis-pyrrole) films Pyrrole coupling chemistry: Investigation of electroanalytic, spectroscopic and thermal properties of N-substituted poly(bis-pyrrole) films. RSC Adv. 2013, 3, 2035-2042.

17. Jang, K.S.; Kim, D.O. Synchronous vapor-phase polymerization of poly(3,4-ethylenedioxythiophene) and poly(3-hexylthiophene) copolymer systems for tunable optoelectronic properties. Org. Electron. 2010, 11, 1668-1675. [CrossRef]

18. Waware, U.S.; Hamouda, A.M.S.; Majumdar, D. Synthesis, characterization and physicochemical studies of copolymers of aniline and 3-nitroaniline. Polym. Bull. 2020, 77, 4469-4488. [CrossRef]

19. Wu, T.M.; Chang, H.L.; Lin, Y.W. Synthesis and characterization of conductive polypyrrole with improved conductivity and processability. Polym. Int. 2009, 58, 1065-1070. [CrossRef]

20. Husson, J.; Lakard, S.; Monney, S.; Buron, C.C.; Lakard, B. Elaboration and characterization of carboxylic acid-functionalized polypyrrole films. Synth. Met. 2016, 220, 247-254. [CrossRef]

21. Sadki, S.; Schottland, P.; Brodie, N.; Sabouraud, G. The mechanisms of pyrrole electropolymerization. Chem. Soc. Rev. 2000, 29, 283-293.

(C) 2020 by the authors. Licensee MDPI, Basel, Switzerland. This article is an open access article distributed under the terms and conditions of the Creative Commons Attribution (CC BY) license (http://creativecommons.org/licenses/by/4.0/). 